\title{
Familial Risks for Diseases of Myoneural Junction and Muscle in Siblings Based on Hospitalizations and Deaths in Sweden
}

\author{
Kari Hemminki, ${ }^{1,2}$ Xinjun Li, ${ }^{2}$ and Kristina Sundquist ${ }^{2}$ \\ ' Division of Molecular Genetic Epidemiology, German Cancer Research Center, Heidelberg, Germany \\ ${ }^{2}$ Center for Family Medicine, Karolinska Institute, Huddinge, Sweden
}

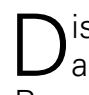
iseases of the myoneural junction and muscle are disabling and some are life-threatening. Recent successes in the identification of the underlying genetic mechanisms have had profound implication for their diagnostics, treatment and classification. We define familial risks for siblings who were hospitalized for or deceased from diseases of the myoneural junction and muscle. A nationwide database on diseases of the myoneural junction and muscle was constructed by linking the Multigeneration Register on 0- to 69-year-old siblings to the Hospital Discharge Register and the Causes of Death Register from years 1987 to 2001. Standardized risk ratios (SIRs) were calculated for affected sibling pairs by comparing to those whose siblings had no diseases of myoneural junction and muscle. Among a total of 2307 patients, myasthenia gravis, muscular dystrophy and myotonic disorders were commonest diagnoses. The sibling risks for these disease were 22, 190 and 198, respectively, when a sibling was diagnosed with any disease of the myoneural junction and muscle. The concordant SIRs, both siblings presenting the same disease, were 42 for myasthenia gravis, 737 for muscular dystrophy, 2000 for congenital myopathy, 1211 for myotonic disorder, 909 for periodic paralysis and 209 for unspecified myopathy. Only a few discordant sibling pairs were noted. The very high overall SIRs for the diseases of the myoneural junction and muscle imply that the sporadic forms of these diseases are relatively rare and these diseases are overwhelmingly heritable.

Diseases of the neuromuscular junction and muscle (myopathies) constitute many individual diseases, which may be characterized by weakness, fatigability, hypercontractility (myotonia), paralysis, pain or atrophy of muscles (Ropper \& Brown, 2005). There has been a remarkable success in the last two decades in the molecular and pathophysiological characterization of these diseases, which has provided diagnostic tests and helped in disease classification (Fokstuen et al., 2001; Muntoni \& Voit, 2004; Upadhyaya \& Cooper, 2002; Voit, 2004). Myastenias are diseases affecting the cholinergic neuromuscular transmission in a rare congenital inherited form through mutations in the structural and functional components of the neuromuscular junction, or in a common acquired form, which is an autoimmune disease with antibodies elicited against proteins at the junction (Harper, 2004; Vincent et al., 2003). Muscular dystrophies are inherited disorders causing progressive muscle weakness and wasting, of which many subtypes of varying age of onset and rate of progression have been distinguished (Day \& Ranum, 2005b; Muntoni \& Voit, 2004; Ropper \& Brown, 2005). Subtypes of muscular dystrophy are characterized by different modes of inheritance and specific mutations in tens of genes, mostly coding for components of the muscle (Dalkilic \& Kunkel, 2003; Emery, 2002). The commonest of these is Duchenne muscular dystrophy, an X-linked disorder affecting preferentially males who carry a mutation in the dystrophin gene. This is among the largest genes known and about $30 \%$ of the affected males carry a de novo mutation and thus lack a family history (Ropper \& Brown, 2005). Duchenne muscular dystrophy and most related muscular dystrophies are diagnosed in the first or second decade of life; however, there are also several congenital and adult forms of muscular dystrophies (Muntoni \& Voit, 2004; Ropper \& Brown, 2005). The latter include myotonic dystrophies 1 and 2, in which the genes are dysregulated by a novel type of mechanism through nucleotide expansions in sequences not translated to protein; the expanded sequences are believed to perturb the function of RNA binding proteins (Day \& Ranum, 2005a; Ranum \& Day, 2004). Nondystrophic myotonic diseases and periodic paralysis are hereditary diseases of muscle membrane excitability, many of which have been traced to mutations in genes coding for ion channels (Ropper \& Brown, 2005). Diverse inflammatory myopathies may result from infections or from an apparent

Received 14 February, 2006; accepted 3 April, 2006.

Address for correspondence: K. Hemminki, DKFZ, Im Neuenheimer Feld 580,D-69120 Heidelberg, Germany.E-mail: k.hemminki@dkfz.de 
autoimmune response (Shamim et al., 2000). Myopathies are also caused by metabolic disturbances of toxic effects of drugs and other chemicals (Ropper \& Brown, 2005).

Many of the diseases of the myoneural junction and muscle are heritable and the genes have been identified in families presenting specific syndromes. The overt heritable patterns in high penetrance forms of these diseases have allowed clinical counseling without data on familial risks at the population level. To our knowledge no population-based family studies of these diseases have been carried out, although data would be available in systematically collected databases on inherited disorders (Al-Jader et al., 2001; Hemminki, Li, \& Sundquist, 2006). The availability of disease-specific data on all hospitalizations and deaths in Sweden prompted us to analyze risks between these diseases with a special motive to observe to what extent these diagnostic systems capture diseases of high heritability, how the familial risks compare, and whether families are found with members diagnosed with diverse subtypes of the diseases of myoneural junction and muscle. Moreover, we wanted to search for familial risks in disease types lacking overt heritability. In the present study we used the Swedish data resources on registered families and hospitalized and fatal cases of diseases of myoneural junction and muscle, both with complete national coverage, to analyze familial risks of diseases of the myoneural junction and muscle between siblings. The Swedish family dataset, the Multigeneration Register, has been validated and extensively used in the study of familial cancer (Hemminki, Li, \& Czene, 2004) and recently on neurological diseases (Hemminki, Li, Johansson, Sundquist, \& Sundquist, 2005, 2006).

\section{Materials and Methods}

The research database used for this study, the neurological database, is a subset of the national MigraMed database at Karolinska Institute, Centre for Family and Community Medicine. The MigMed database was compiled using data from several national Swedish registers provided by Statistics Sweden, the Swedish government-owned statistics bureau; the Multigeneration Register in which persons (second generation) born in Sweden in 1932 or thereafter are registered shortly after birth and are linked to their parents (first generation). Sibships could only be defined for the second generation, which was the present study population. National Census Data (1960-1990) and the Swedish population register (1990-2001) were incorporated into the database to obtain information on individuals' socioeconomic status. Dates of hospitalization for diseases of the myoneural junction and muscle were obtained during the study period from the Swedish Hospital Discharge Register (included into the MigMed database). Since 1986, complete data on all discharges, with dates of hospitalization and diagnoses, have been recorded in this register. Patients who had died from these diseases were identified from the Cause of Death Register and included in the analysis.

All linkages were performed using the national 10-digit civic identification number that is assigned to each person in Sweden for his or her lifetime. This number was replaced by a serial number for each person in order to provide anonymity. The serial number was used to check that each individual was only entered once, for his or her first appearance with diseases of myoneural junction and muscle diagnosis. Over 6.9 million individuals were included in the second generation of the neurological database.

\section{Outcome Variable}

Patients with diseases of the myoneural junction and muscle were retrieved from hospital discharge notifications. All patients registered for hospitalization stayed at least one night in the hospital, usually in wards with specialist consultation or neurology departments; the Register does not include outpatients in hospitals or healthcare centers. Diagnoses were reported according to the 9th (1987-1996) and 10th (1997-2001) versions of the International Classification of Diseases (ICD; World Health Organization, 1977, 2004). The subtypes of diseases of myoneural junction and muscle and the number of hospitalized patients are shown in Table 1 . For some analysis, we combined the codes of ICD-9 and ICD-10 when they were identical in the two classification systems. A total of 2307 patients were identified with disease of myoneural junction and muscle, of whom 2201 from the Hospital Discharge Register and 106 $(4.6 \%)$ from the Cause of Death Register.

\section{Individual Variables}

Gender was categorized as men and women. Age at diagnosis was categorized as follows: $0-4,5-9$, $10-14, \ldots 60-64,65-69$ years. The men's and women's socioeconomic status was divided into six groups according to occupation or, for those without occupation (e.g., housewives), their husband's/ father's occupation: (1) farmers, (2) unskilled workers, (3) blue collar workers, (4) professionals, (5) selfemployed people, and (6) all others. Many of the present diseases are highly hereditable and the socioeconomic status is not likely to be of importance; however, for acquired forms of the diseases it may have an effect. Nevertheless, Sweden has free access to hospitalization regardless of personal socioeconomic status.

Geographical region was divided into three groups: (1) big cities (cities with a population of over 200,000, i.e., Stockholm, Gothenburg, and Malmö), (2) Southern, and (3) Northern Sweden, the dividing line being the Dala river. Geographical region was included in order to adjust for possible differences between regions regarding the access to specialized hospital care. 


\section{Statistical Analysis}

Person-years were calculated from start of follow-up on January 1, 1987 until hospitalization for diseases of the myoneural junction and muscle, death, emigration, or closing date, December 31, 2001. Age-specific incidence rates were calculated for the whole follow-up period, divided into five 5-year periods. Standardized incidence ratios (SIRs) were calculated as the ratio of observed $(\mathrm{O})$ to expected $(\mathrm{E})$ number of cases. The expected number of cases was calculated for age (5-year groups), sex, period (5-year groups), region and socioeconomic status-specific standard incidence rates. Sibling risks were calculated for men and women with siblings affected with diseases of the myoneural junction and muscle, compared with men and women whose siblings were not affected by these conditions, using the cohort method as described (Hemminki et al., 2001). We defined a cohort of individuals with at least one affected sibling and computed the incidence rates in this cohort over the study period. In a family with two or more affected siblings, each affected individual is included in the cohort (as the sibling of an affected individual). The incidence rates in the cohort method are given by the formula:

$$
\frac{\sum_{k=2}^{N} n_{k}}{\left[\sum_{k=1}^{N} p_{k}+\sum_{k=2}^{N} y_{k}\right]}
$$

where $n_{k}$ is the number of affected individuals with $k$ affected siblings, $p_{k}$ the number of person-years contributed by unaffected individuals in families with $k$ affected siblings, and $y_{k}$ the number of person-years contributed by affected siblings in families with $k$ affected siblings, in the relevant age/sex/period/ region/socioeconomic status category. The corresponding reference rates are given by:

$$
\frac{\sum_{k=1}^{N} n_{k}}{\left[\sum_{k=0}^{N} p_{k}+\sum_{k=1}^{N} y_{k}\right]}
$$

Confidence intervals (95\% CI) were calculated assuming a Poisson distribution, and they were adjusted for dependence between the sibling pairs (Hemminki et al., 2001). Lower limits of $95 \%$ CIs were quoted as 1 (Newcombe, 1988).

\section{Results}

A total of 2307 unique patients were hospitalized for or died from diseases of the myoneural junction and muscle in Sweden at ages 0 to 69 years between years 1987 and 2001 (Table 1). Men accounted for 54.4\% of the patients (data not shown). According to ICD-9, myasthenia gravis and hereditary progressive muscular dystrophy each accounted for a quarter of all cases, followed by unspecified myopathy and myotonic disorders. According to ICD-10, myasthenia gravis and muscular dystrophy were the commonest diagnoses. A total of 182 siblings were diagnosed with diseases of the myoneural junction and muscle, constituting $7.9 \%$ of all affected individuals. Very high familial risks were recorded, according to ICD-9, for congenital hereditary muscular dystrophy (SIR 254), hereditary progressive muscular dystrophy (190), myotonic disorders (198), familial periodic paralysis (202) and unspecified myopathies (131). High risks were noted also for myasthenia gravis (22) and other myopathies (21). On the other hand, no cases were observed for toxic, endocrine-disease or inflammatory myopathies. The overall SIR for diseases of the myoneural junction and muscle was 149. The overall SIR was essentially similar, 106, according to ICD-10. Myotonic disorders showed the highest SIR of 1154, clearly higher than the SIR according to ICD-9.

Familial risks shown in Table 1 were analyzed separately for men and women and their overall SIRs did not differ; however, because of the small number we show no results by gender. According to ICD-9, the SIR for hereditary progressive muscular dystrophy in men was $225(N=41,95 \%$ CIs 114-432) and in women it was $121(11,43-308)$. According to ICD-10 for muscular dystrophy, these were 280 (12, 102-693) and 91 (4, 17-334), respectively.

In Table 2 we show familial risks when the siblings presented with the concordant (same) or discordant (different) disease of the myoneural junction and muscle, based on the merged ICD-9 and ICD-10 codes. Concordant SIRs were always the highest and they exceeded the SIRs from Table 1. The concordant SIRs were 42 for myasthenia gravis, 737 for muscular dystrophy, 2000 for congenital myopathy, 1211 for myotonic disorder, 909 for periodic paralysis and 209 for unspecified myopathy. Most discordant associations were with unspecified myopathies. However, muscular dystrophy and myotonic disorders also associated with each other with SIRs of 139 and 170.

\section{$\overline{\text { Discussion }}$}

In the present study we used SIR as a measure of familial risk between siblings, expressing the likelihood of an individual being affected, given a diagnosed disease of the myoneural junction or muscle in a sibling. These are rare diseases and the number of affected sibling pairs was limited; furthermore, only diseases leading to hospitalization before age 69 years were included. The national Hospital Discharge Register has been operated only since 1987 , the present study covered a time period of no longer than 15 years and thus pedigrees would not be very informative. The relatively short follow-up time also limits the number of observed affected sibling pairs. However, because the person-years at risk are 
Table 1

Total Number of Hospitalized and Deceased Individuals With Diseases of Myoneural Junction and Muscle According to ICD-9 and ICD-10 and Sibling History When a Sibling Was Diagnosed With Any of These Diseases

\begin{tabular}{|c|c|c|c|c|}
\hline \multirow[t]{2}{*}{ ICD code, subtype } & \multicolumn{2}{|c|}{ All cases } & \multicolumn{2}{|c|}{ Sibling history } \\
\hline & Cases & $\%$ & Observed & Expected \\
\hline \multicolumn{5}{|l|}{ ICD-9, 1987-1996 } \\
\hline 358.A, myasthenia gravis & 404 & 25.3 & 4 & 0.2 \\
\hline 358.B, myasthenic syndromes in diseases classified elsewhere & 4 & 0.3 & 0 & 0.0 \\
\hline 358.C, toxic myoneural disorders & 3 & 0.2 & 0 & 0.0 \\
\hline 358.W, other specified myoneural disorders & 5 & 0.3 & 0 & 0.0 \\
\hline 358.X, myoneural disorders, unspecified & 47 & 2.9 & 1 & 0.0 \\
\hline 359.A, congenital hereditary muscular dystrophy & 79 & 5.0 & 9 & 0.0 \\
\hline 359.B, hereditary progressive muscular dystrophy & 378 & 23.7 & 52 & 0.3 \\
\hline 359.C, myotonic disorders & 252 & 15.8 & 47 & 0.2 \\
\hline 359.D, familial periodic paralysis & 76 & 4.8 & 7 & 0.0 \\
\hline 359.E, toxic myopathy & 21 & 1.3 & 0 & 0.0 \\
\hline 359.F, myopathy in endocrine diseases classified elsewhere & 4 & 0.3 & 0 & 0.0 \\
\hline 359.G, symptomatic inflammatory myopathy in diseases classified elsewhere & 13 & 0.8 & 0 & 0.0 \\
\hline 359.W, other myopathies & 37 & 2.3 & 2 & 0.1 \\
\hline 359.X, myopathy, unspecified & 272 & 17.1 & 19 & 0.1 \\
\hline All & 1595 & 100.0 & 141 & 0.9 \\
\hline \multicolumn{5}{|l|}{ ICD-10, 1997-2001 } \\
\hline G70.0, myasthenia gravis & 173 & 24.3 & 0 & 0.1 \\
\hline G70.1, toxic myoneural disorders & 1 & 0.1 & 0 & 0.0 \\
\hline G70.2, congenital and developmental myasthenia & 3 & 0.4 & 0 & 0.0 \\
\hline G70.8, other specified myoneural disorders & 7 & 1.0 & 2 & 0.0 \\
\hline G70.9, myoneural disorders, unspecified & 24 & 3.4 & 0 & 0.0 \\
\hline G71.0, muscular dystrophy & 133 & 18.7 & 16 & 0.1 \\
\hline G71.1, myotonic disorders & 119 & 16.7 & 18 & 0.0 \\
\hline G71.2, congenital myopathies & 27 & 3.8 & 2 & 0.0 \\
\hline G71.3, mitochondrial myopathy, not elsewhere classified & 33 & 4.6 & 1 & 0.0 \\
\hline G71.8, other primary disorders of muscles & 6 & 0.8 & 1 & 0.0 \\
\hline G71.9, primary disorder of muscle, unspecified & 60 & 8.4 & 0 & 0.0 \\
\hline G72.0, drug-induced myopathy & 8 & 1.1 & 0 & 0.0 \\
\hline G72.1, alcoholic myopathy & 4 & 0.6 & 0 & 0.0 \\
\hline G72.3, myopathy due to other toxic agents & 13 & 1.8 & 0 & 0.0 \\
\hline G72.4, inflammatory myopathy, not elsewhere classified & 6 & 0.8 & 0 & 0.0 \\
\hline G72.8, other specified myopathies & 6 & 0.8 & 0 & 0.0 \\
\hline G72.9, myopathy, unspecified & 86 & 12.1 & 1 & 0.0 \\
\hline G73, disorders of myoneural junction and muscle in diseases classified elsewhere & 3 & 0.4 & 0 & 0.0 \\
\hline All & 712 & 100.0 & 41 & 0.4 \\
\hline
\end{tabular}

Note: ICD = International Classification of Diseases

similarly censored, the effects on familial risk estimates are not large, but they would be underestimates. The remarkably similar familial risk estimates on multiple sclerosis from the current database and from the Danish Multiple Sclerosis Register testify for the current method of analysis and the diagnostic accuracy (Hemminki, Li, Johansson, et al., 2006; Nielsen et al., 2005). Another limitation of the present study is that it covered a period of large diagnostic improvements in neurological diseases and, being a nationwide study, the diagnostic accuracy could be have varied within the study period and within the country. However, overall the diagnostic accuracy was probably good as severe conditions require repeated hospitalizations, which normally involve diagnosis by several physicians, including neurologists (Johansson \& Westerling, 2000). Moreover, any errors in diagnosis would have reduced the concordance between sibling pairs, which was remarkable in Table 2. On the other hand, for the congenital forms of the present diseases a remote possibility 
exists that a known diagnosis in one sibling may influence the diagnosis for the other sibling. The available code system did not allow a closer specification of the diseases, which would be helpful for genetic diseases (Al-Jader et al., 2002).

The present study, including only hospitalized and deceased cases of diseases of the myoneural junction and muscle, showed very high overall familial risks of over 100, implying that an individual with an affected sibling was 100 times more likely to be diagnosed with these diseases than individuals whose siblings did not suffer from these diseases. Being heritable diseases, the risks were primarily for one type of the disease, as shown in Table 2. All the main types, muscular dystrophy, congenital myopathy, myotonic disorders and periodic paralysis showed concordant familial risk of the order of 1000. In muscular dystrophy the SIR was two to three times higher for men than for women, consistent with the preferential male risk for the common X-linked subtypes of Duchenne/ Becker and Emery/Dreifuss (Dalkilic \& Kunkel, 2003; Emery, 2002; Muntoni \& Voit, 2004). Discordant associations were mainly between specific and unspecific types, which may be due to diagnostic practices, that is, hesitation to use a specific diagnosis in uncertain cases, particularly in the early part of the study period when the diagnostic tests were not available. Similarly, muscular dystrophy and myotonic disorders associated with each other but the discordant cases were few compared to concordant cases. Many of the current diagnostic tests were not available in the early study period, and the much higher SIR for myotonic disorders, 1154 in the last period (1997-2001) compared to 198 the first period (1987-1996) is likely to be due to the sophistication of diagnostic procedures. Myasthenia gravis, with a familial risk of 42, showed a low familial risk among this group of diseases. The sporadic autoimmune forms of this disease are by far more common than the heritable forms (Harper, 2004). However, the present data on a familial risk of 42 may imply a considerable familial clustering even for the autoimmune form of the disease. Previously, the present data sources have been extensively to study familial cancers. For comparison, the common forms of cancer show overall sibling risks of around two, and risks of greater than 100 have only been observed for some rare heritable conditions such as medullary thyroid cancer in multiple endocrine neoplasia 2 and hemangioblastoma in von Hippel-Lindau disease (Hemminki, Rawal, et al., 2004).

Diseases of the myoneural junction and muscle also contained subtypes of apparent environmental etiology, such as toxic myoneural disorders, and various toxic and inflammation-related myopathies, because no familial cases were found. However, these conditions were rare and only high risks 
would have been recognized. The overwhelmingly high overall SIRs nevertheless imply that the sporadic forms of diseases of the myoneural junction and muscle are relatively rare and these diseases have mainly a heritable etiology. The remarkable success in the past 20 years to characterize gene defects for the various subtypes of these diseases is an outstanding success story of molecular medicine (Day \& Ranum, 2005b; Muntoni \& Voit, 2004; Ropper \& Brown, 2005). The present technologies are capable of identifying genes for monogenic forms of disease. An interesting question, beyond the present approach, is whether the heritable mechanisms underlying the diseases of the myoneural junction and muscle have been largely unraveled or whether novel discoveries are yet to be expected.

In conclusion, this nationwide study on medically diagnosed cases of diseases of the myoneural junction and muscle show very high familial risks for the diseases of known high heritability, implying that sporadic forms of these diseases are rare. The specificity of the familial risks also attests to the monogenic origin of these disease forms. On the other hand, for toxicity related myopathies no familial cases were observed, but because of their rarity only high risks could be excluded. The study demonstrates the feasibility of nationwide family studies of major hospitalized diseases.

\section{Acknowledgments}

This work was supported by grants from the National Institutes of Health (Grant No. R01H271084-1), the Swedish Research Council (Grant No. K2004-21X-11651-09A to Dr Jan Sundquist and K2005-27X-15428-01A to Dr Kristina Sundquist), and the Swedish Council for Working Life and Social Research (Grant No. 2001-2373 and 2005-0039).

\section{References}

Al-Jader, L. N., Harper, P. S., Krawczak, M., Palmer, S. R., Johansen, B. N., \& Cooper, D. N. (2001). The frequency of inherited disorders database. Human Genetics, 108, 72-74.

Al-Jader, L. N., Newcombe, R. G., Hayes, S., Murray, A., Layzell, J., \& Harper, P. S. (2002). Developing a quality scoring system for epidemiological surveys of genetic disorders. Clinical Genetics, 62, 230-234.

Dalkilic, I., \& Kunkel, L. M. (2003). Muscular dystrophies: Genes to pathogenesis. Current Opinion in Genetics and Development, 13, 231-238.

Day, J. W., \& Ranum, L. P. (2005a). Genetics and molecular pathogenesis of the myotonic dystrophies. Current Neurology and Neuroscience Reports, 5, 55-59.

Day, J. W., \& Ranum, L. P. (2005b). RNA pathogenesis of the myotonic dystrophies. Neuromuscular Disorders, 15, 5-16.
Emery, A. (2002). The muscular dystropies. Lancet, 359, 687-695.

Fokstuen, S., Myring, J., Evans, C., \& Harper, P. S. (2001). Presymptomatic testing in myotonic dystrophy: Genetic counselling approaches. Journal of Medical Genetics, 38, 846-850.

Harper, C. (2004). Congenital myastenic syndromes. Seminars in Neurology, 24, 111-123.

Hemminki, K., Li, X., \& Czene, K. (2004). Familial risk of cancer: Data for clinical counseling and cancer genetics. International Journal of Cancer, 108, 109-114.

Hemminki, K., Li, X., Johansson, S., Sundquist, K., \& Sundquist, J. (2005). Familial risks for migraine and other headaches among siblings based on hospitalisations in Sweden. Neurogenetics, 6, 217-224.

Hemminki, K., Li, X., Johansson, S., Sundquist, K., \& Sundquist, J. (2006). Re. Familial risk of multiple sclerosis: A nationwide cohort study. American Journal of Epidemiology, 163, 873-874.

Hemminki, K., Rawal, R., Chen, B., \& Lorenzo Bermejo, J. (2004). Genetic epidemiology of cancer: From families to heritable genes. International Journal of Cancer, 111, 944-950.

Hemminki, K., Sundquist, K., \& Li, X. (2006). Familial risks for main neurological diseases in siblings based on hospitalizations in Sweden. Twin Research and Human Genetics, 9, 580-586.

Hemminki, K., Vaittinen, P., Dong, C., \& Easton, D. (2001). Sibling risks in cancer: Clues to recessive or X-linked genes? British Journal of Cancer, 84, 388-391.

Johansson, L. A., \& Westerling, R. (2000). Comparing Swedish hospital discharge records with death certificates: Implications for mortality statistics. International Journal of Epidemiology, 29, 495-502.

Muntoni, F., \& Voit, T. (2004). The congenital muscular dystrophies in 2004: A century of exciting progress. Neuromuscular Disorders, 14, 635-649.

Newcombe, R. (1988). Two-sided confidence intervals for thr single proportion: Comparison of seven methods. Statistics in Medicine, 17, 857-872.

Nielsen, N. M., Westergaard, T., Rostgaard, K., Frisch, M., Hjalgrim, H., Wohlfahrt, J., Koch-Henriksen, N., \& Melbye, M. (2005). Familial risk of multiple sclerosis: A nationwide cohort study. American Journal of Epidemiology, 162, 1-5.

Ranum, L. P., \& Day, J. W. (2004). Pathogenic RNA repeats: An expanding role in genetic disease. Trends in Genetics, 20, 506-512.

Ropper, A., \& Brown, R. (2005). Adams and Victor's principles of neurology (8th ed.). New York: McGraw-Hill.

Shamim, E. A., Rider, L. G., \& Miller, F. W. (2000). Update on the genetics of the idiopathic inflammatory 
myopathies. Current Opinion in Rheumatology, 12, 482-491.

Upadhyaya, M., \& Cooper, D. N. (2002). Molecular diagnosis of facioscapulohumeral muscular dystrophy. Expert Review of Molecular Diagnostics, 2, 160-171.

Vincent, A., McConville, J., Farrugia, M. E., Bowen, J., Plested, P., Tang, T., Evoli, A., Matthews, I., Sims, G., Dalton, P., Jacobson, L., Polizzi, A., Blaes, F., Lang, B., Beeson, D., Willcox, N., Newsom-Davis, J., \& Hoch, W. (2003). Antibodies in myasthenia gravis and related disorders. Annals of the New York Academy of Sciences, 998, 324-335.

Voit, T. (2004). The pediatric neurologist of the 21st centrury needs an encyclopedic mind. Neuropediatrics, 35, 319-320.

World Health Organization. (1977). International classification of diseases (ICD-9, 9th ed.). Geneva: WHO Press.

World Health Organization. (2004). International statistical classification of diseases and health related problems (ICD-10, 2nd ed.). Geneva: WHO Press. 